\title{
A UNIFORMLY CONVEX BANACH SPACE WITH A SCHAUDER BASIS WHICH IS SUBSYMMETRIC BUT NOT SYMMETRIC
}

\author{
LAKHPAT R. PUJARA
}

AbSTRACT. An example of a uniformly convex Banach space with a basis $\left(x_{i}\right)$ is contructed such that $\left(x_{i}\right)$ is subsymmetric but not symmetric.

1. Introduction. Garling [3], [4] gave an example of a nonuniformly convex Banach space with a basis which is subsymmetric but not symmetric. Professor Bill Johnson has conveyed to us that Dacunha Castelle has recently proved that in $L_{p}([0,1]), 1<p<\infty$, every basic sequence which is subsymmetric is also symmetric. Of course, it is well known that $L_{p}([0,1]), 1 \leqslant p$ $<\infty, p \neq 2$, has no subsymmetric basis. In this paper, a uniformly convex Banach space with a basis $\left(\delta_{i}\right)$ is constructed such that $\left(\delta_{i}\right)$ is subsymmetric but not symmetric. This substantiates a conjecture of William B. Johnson.

We follow the standard notation for Banach space theory. A basis $\left(x_{n}\right)$ for a space $(X,\|\cdot\|)$ is called unconditionally monotone if $\left\|\sum \alpha_{n} x_{n}\right\| \leqslant\left\|\sum \beta_{n} x_{n}\right\|$ whenever $\left|\alpha_{n}\right| \leqslant\left|\beta_{n}\right|$. Suppose $X$ has an unconditionally monotone basis $\left(x_{i}\right)$. The norm on $X$ is called $p$-convex if

$$
\left\|\Sigma\left(\left|\alpha_{i}\right|^{p}+\left|\beta_{i}\right|^{p}\right)^{1 / p} x_{i}\right\|^{p} \leqslant\left\|\sum \alpha_{i} x_{i}\right\|^{p}+\left\|\Sigma \beta_{i} x_{i}\right\|^{p}, \quad p \geqslant 1,
$$

for all scalars $\left(\alpha_{i}\right)$ and $\left(\beta_{i}\right)$. The norm on $X$ is said to satisfy an upper $l_{p}$-estimate if $\|x+y\|^{p} \leqslant\|x\|^{p}+\|y\|^{p}$ whenever $x$ and $y$ have disjoint support relative to $\left(x_{i}\right)$. It is clear that if $\|\cdot\|$ is $p$-convex, then it satisfies the upper $l_{p}$-estimate. The norm on $X$ is said to satisfy a lower $l_{q}$-estimate, if $\|x+y\|^{q} \geqslant\|x\|^{q}+\|y\|^{q}$ whenever $x$ and $y$ have disjoint support relative to $\left(x_{i}\right)$. A basis $\left(x_{i}\right)$ is called subsymmetric if it is unconditional and equivalent to each of its subsequences. It is called symmetric if it is equivalent to each of its permutations.

The author would like to thank Professor William Johnson and Professor Ranko Bojanic for several useful conversations regarding this paper.

2. The construction of the space. Let $X$ denote the space of all scalar valued sequences which have only finitely many nonzero coordinates. As in [4], we define a norm on $X$ by

$$
\left\|\left(\beta_{n}\right)\right\|=\left(\sup _{n_{1}<n_{2}<\ldots} \ldots \sum_{i} \frac{\beta_{n_{i}}^{2}}{\sqrt{i}}\right)^{1 / 2} .
$$

Received by the editors April 16, 1975.

AMS (MOS) subject classifications (1970). Primary 46B99.

Key words and phrases. Uniformly convex space, subsymmetric basis, symmetric basis. 
It follows immediately that the norm $\|\cdot\|$ is 2-convex. From now on, let $r=4$, 8. We define two other norms on $X$ as follows:

$$
\left|\left(\beta_{n}\right)\right|_{r}=\left(\sup _{\left(A_{k}\right)} \sum_{k}\left\|\sum_{i \in A_{k}} \beta_{i} \delta_{i}\right\|^{r}\right)^{1 / r}
$$

where the $A_{k}$ 's are pairwise disjoint finite subsets of the integers and $\left(\delta_{i}\right)$ are unit vectors. It is a routine calculation to show that $\left(X,|\cdot|_{r}\right)$ is a Banach space.

TheOREM. The space $\left(X,|\cdot|_{8}\right)$ is a uniformly convex Banach space. The unit vectors $\left(\delta_{i}\right)$ constitute a subsymmetric basis for $X$ which is not symmetric.

Proof. We divide the proof into several steps.

Step I. It is straightforward to show that $\left(\delta_{i}\right)$ is an unconditionally monotone basis for $X,\left(\delta_{i}\right)$ is subsymmetric and that $\left.\right|_{r}$ satisfies the lower $l_{r}$-estimate.

Step II. We assert that the norm $|\cdot|_{r}$ is 2-convex. Indeed, let

$$
\left|\sum_{i}\left(\left|\alpha_{i}\right|^{2}+\left|\beta_{i}\right|^{2}\right)^{1 / 2} \delta_{i}\right|^{2}=\left[\sum_{k=1}^{j}\left\|\left(\left|\alpha_{i}\right|^{2}+\left|\beta_{i}\right|^{2}\right)^{1 / 2} \delta_{i}\right\|^{r}\right]^{2 / r}
$$

for some pairwise disjoint finite subsets $A_{1}, A_{2}, \ldots, A_{j}$.

$$
\begin{aligned}
& =\left[\sum_{k=1}^{j}\left(\left\|\sum_{i \in A_{k}}\left(\left|\alpha_{i}\right|^{2}+\left|\beta_{i}\right|^{2}\right)^{1 / 2} \delta_{i}\right\|^{2}\right]^{r / 2}\right]^{2 / r} \\
& \leqslant\left[\sum_{k=1}^{j}\left(\left\|\sum_{i \in A_{k}} \alpha_{i} \delta_{i}\right\|^{2}+\left\|\sum_{i \in A_{k}} \beta_{i} \delta_{i}\right\|^{2}\right)^{r / 2}\right]^{2 / r}
\end{aligned}
$$

as $\|\cdot\|$ is 2-convex,

$$
\leqslant\left[\sum_{k=1}^{j}\left(\left\|\sum_{i \in A_{k}} \alpha_{i} \delta_{i}\right\|^{2}\right)^{r / 2}\right]^{2 / r}+\left[\sum_{k=1}^{j}\left(\left\|\sum_{i \in A_{k}} \beta_{i} \delta_{i}\right\|^{2}\right)^{r / 2}\right]^{2 / r}
$$

as $r=4,8$,

$$
\leqslant\left|\sum_{i} \alpha_{i} \delta_{i}\right|_{r}^{2}+\left|\sum_{i} \beta_{i} \delta_{i}\right|_{r}^{2}
$$

It follows that $|\cdot|_{r}$ is 2-convex.

Step. III. Since $\left(X,|\cdot|_{8}\right)$ is 2 -convex and satisfies the lower $l_{8}$-estimate, it is uniformly convex, using a theorem of Figiel and Johnson [2].

Step IV. We will now show that $\left(\delta_{i}\right)$ is not symmetric. For $k=2^{m}, m=0$, $1,2, \ldots$, define

$$
y_{m}=\left(k^{-1 / 4},(k-1)^{-1 / 4}, \ldots, 2^{-1 / 4}, 1,0,0, \cdots\right)
$$

and 


$$
\bar{y}_{m}=\left(1,2^{-1 / 4}, 3^{-1 / 4}, \ldots,(k-1)^{-1 / 4}, k^{-1 / 4}, 0, \cdots\right) .
$$

It is enough [4] to show that

$$
\left|\bar{y}_{m}\right|_{8} /\left|y_{m}\right|_{8} \rightarrow \infty \quad \text { as } m \rightarrow \infty \text {. }
$$

Clearly

$$
\begin{aligned}
\left|\bar{y}_{m}\right|_{8} & \geqslant\left(1+2^{-1 / 2} \cdot 2^{-1 / 2}+\cdots+k^{-1 / 2} \cdot k^{-1 / 2}\right)^{1 / 2} \\
& \geqslant\left(c_{1}+m \cdot \log 2\right)^{1 / 2}
\end{aligned}
$$

for large $m, c_{1}$ being a constant.

Now we use $|\cdot|_{4}$ to obtain an upper estimate for $\left|y_{m}\right|_{8}$. Using the 2convexity of $|\cdot|_{4}$, we get

$$
\left|y_{m}\right|_{4}^{2} \leqslant \sum_{j=1}^{m+1}\left|z_{j}\right|_{4}^{2}
$$

where

$$
\begin{gathered}
z_{j}=\left(0,0, \ldots,\left(2^{m+1-j}\right)^{-1 / 4},\left(2^{m+1-j}-1\right)^{-1 / 4}, \ldots,\left(2^{m-j}+1\right)^{-1 / 4}, 0, \cdots\right), \\
1 \leqslant j \leqslant m, \\
z_{m+1}=(0,0, \ldots, 1,0, \cdots),
\end{gathered}
$$

and

$$
\operatorname{supp}\left(z_{j}\right) \cap \operatorname{supp}\left(z_{l}\right)=\varnothing, \quad j \neq l .
$$

To find an upper estimate for $\left|z_{j}\right|_{4}$, for the sake of convenience of notation, consider

$$
x_{l}=\left(0,0, \ldots,(2 l)^{-1 / 4},(2 l-1)^{-1 / 4}, \ldots,(l+1)^{-1 / 4}, 0, \cdots\right)
$$

where $l$ is an integer. Let the norm of $x_{l}$ be attained over the pairwise disjoint finite subsets $A_{1}, A_{2}, \ldots, A_{s}$. Let

$$
A_{n}=\left\{i_{1}^{(n)}, i_{2}^{(n)}, \ldots, i_{r_{n}}^{(n)}\right\}, \quad i_{1}^{(1)}=1,1 \leqslant n \leqslant s .
$$

Using an argument as in [4, p. 586] and the fact that $\left(A_{n}\right)_{n=1}^{s}$ gives the norm for $x_{l}$, we have $\sum_{i=1}^{s} r_{i}=l$ and

$$
\begin{aligned}
\left|x_{l}\right|_{4}^{4} & =\sum_{n=1}^{s}\left[\sum_{n=1}^{r_{n}} \frac{1}{\sqrt{2 l+1-i_{p}^{(n)}}} \cdot \frac{1}{\sqrt{p}}\right)^{2} \\
& \leqslant \frac{1}{l+1} \cdot \sum_{n=1}^{s}\left(\sum_{p=1}^{r_{n}} \frac{1}{\sqrt{p}}\right)^{2} \\
& \leqslant \frac{1}{l+1} \cdot \sum_{n=1}^{s} 4 \cdot r_{n}=\frac{4}{l+1} \cdot l \leqslant 4
\end{aligned}
$$

i.e.

$$
\left|x_{l}\right|_{4}^{2} \leqslant 2
$$


Using (3) in (2), we get

$$
\left|y_{m}\right|_{4} \leqslant \sqrt{2(m+1)} .
$$

It can be proved, as in [4], that

$$
\left\|y_{m}\right\| \leqslant c_{2}
$$

where $c_{2}$ is a constant.

Let $\left(B_{i}\right)_{i=1}^{s}$ be any pairwise disjoint finite subsets of the integers. For the sake of convenience, write $y_{m}=\sum \alpha_{i} \delta_{i}$. Then we have

$$
\sum_{j=1}^{s}\left\|\sum_{i \in B_{j}} \alpha_{i} \delta_{i}\right\|^{8} \leqslant\left\|y_{m}\right\|^{4} \cdot \sum_{j=1}^{s}\left\|\sum_{i \in B_{j}} \alpha_{i} \delta_{i}\right\|^{4}
$$

i.e.

$$
\left(\sum_{j=1}^{s}\left\|\sum_{i \in B_{j}} \alpha_{i} \delta_{i}\right\|^{8}\right)^{1 / 8} \leqslant\left(c_{2}\right)^{1 / 2} \cdot\left(\left|y_{m}\right|_{4}\right)^{1 / 2}
$$

using (5),

$$
\leqslant\left(c_{2}\right)^{1 / 2}(2(m+1))^{1 / 4}
$$

using (4).

Since $\left(B_{i}\right)_{i=1}^{s}$ are arbitrary, we get

$$
\left|y_{m}\right|_{8} \leqslant c_{3} \cdot(m+1)^{1 / 4} .
$$

From (1) and (6), we get $\left|\bar{y}_{m}\right|_{8} /\left|y_{m}\right|_{8} \rightarrow \infty$ as $m \rightarrow \infty$. Q.E.D.

\section{REFERENCES}

1. N. Dunford and J. T. Schwartz, Linear operators. I: General theory, Pure and Appl. Math., vol. 7, Interscience, New York, 1958. MR 22 \#302.

2. T. Figiel and W. B. Johnson, $A$ uniformly convex Banach space which contains no $l_{p}$, Compositio Math. 29(1974), 1-12.

3. D. J. H. Garling, Symmetric bases of locally convex spaces, Studia Math. 30(1968), 163-181. MR 37 \#670.

4. I. Singer, Bases in Banach spaces. I, Die Grundlehren der math. Wissenschaften, Band 154, Springer-Verlag, Berlin and New York, 1970. MR 45 \#451.

Department of Mathematics, Ohio State University, Columbus, Ohio 43210

Current address: Department of Mathematics, Wilberforce University, Wilberforce, Ohio 45384 